CASE REPORT

\title{
Candida albicans peritonitis in a patient with Felty's syndrome
}

\author{
D W Thomas, P Munuswamy, K Adu-Poku, C S Holgate, P Hickling, A Lambert, A G Prentice
}

J Clin Pathol 2005;58:432-433. doi: 10.1136/icp.2004.016287

A 53 year old man with Felty's syndrome presented with abdominal pain and fever. He underwent a laparotomy after starting broad spectrum antibiotics. An intestinal biopsy showed skip ulcers with fungal hyphae. Peritoneal exudates grew Candida albicans. He was started on intravenous fluconazole and then switched to liposomal amphotericin to which he showed a good clinical response. After one month at home he was readmitted with candidosis and died of a myocardial infarction.

A 53 year old man presented with abdominal pain lasting one week. The pain started in the epigastrium, localising to the right iliac fossa. He had fever and rigors and complained of vomiting and diarrhoea for four days. The oropharynx appeared to be normal.

Rheumatoid arthritis was diagnosed 10 years previously, with uncomplicated Felty's syndrome for three years. His median neutrophil count during this time ranged from 0.7 to $1.6 \times 10^{9}$ /itre. He also had asthma and ischaemic heart disease and was taking aspirin, diclofenac, amoxicillin, digoxin, and salbutamol and becotide inhalers.

On presentation his neutrophil count was zero. He was started on ceftazidime and gentamicin. His fever continued to spike and he developed diffuse abdominal tenderness. Computed tomography of the abdomen showed hepatosplenomegaly. The pancreas, kidneys, adrenals, and bowel were all normal.

Because he remained unwell, antibiotics were changed to meropenem and vancomycin and a laparotomy was performed. At surgery, skip lesions were seen in the small bowel. A section of small bowel was removed. Purulent peritoneal fluid was cultured.

Blood cultures were negative, but peritoneal fluid grew Candida albicans, sensitive to fluconazole and amphotericin. Oropharyngeal candidiasis was then noted.

Histopathological examination of the ulcerated intestinal mucosa showed fungal hyphae and spores (fig 1) with Gram positive cocci.

Fluconazole was started for the candida infection, and because the fever did not settle, liposomal amphotericin was added. After a week the fever started to abate. His neutrophil count ranged from 0 to $15.6 \times 10^{9}$ /itre without granulocyte colony stimulating factor. After two weeks of Ambisome all intravenous antimicrobials were stopped and oral fluconazole was started at $200 \mathrm{mg}$ daily. He was well when discharged home, without fever and with a neutrophil count of $2.1 \times 10^{9}$ /itre.

One month later he was readmitted with a neutrophil count of zero. Gross oropharyngeal candidosis and signs of abdominal sepsis were noted. Fluconazole had been discontinued in the community two weeks before admission. His abdominal wound had dehisced and was partially necrotic. Methicillin resistant Staphyloccus aureus was cultured from a wound swab. His clinical condition deteriorated despite treatment with amphotericin and vancomycin. He died of a myocardial infarction within two weeks of readmission.

\section{DISCUSSION}

Felty described a syndrome in 1924 consisting of rheumatoid arthritis, splenomegaly, and neutropenia. ${ }^{1}$ There is a high incidence of severe infection associated with this syndrome, with a $66 \%$ overall five year survival. ${ }^{2}$

This increased risk of serious infection is greater when the neutrophil count falls below $1 \times 10^{9}$ /litre. In one of the larger reported series of this complication there were 12 major and 32 minor infections over a three year period in 15 patients. ${ }^{3}$ Only two of the minor episodes were superficial $C$ albicans infection. Systemic fungal infections were not seen. Invasive epiglottitis with $C$ glabrata has been described in a patient with Felty's syndrome. ${ }^{4}$

Phagocytosis by neutrophils is an important part of the host defences against infection with Candida spp, which exist in three forms, namely: yeast/spores, elongated forms without hyphae (pseudohyphae), and true hyphae with septa. The transition from yeast to hyphae is important for fungal virulence. ${ }^{5}$ Hyphae appear able to exit from the cells that engulf them and avoid phagocytic death. The mucosae of the mouth, gut, and vagina may be colonised in up to $80 \%$ of normal individuals. When host defences are compromised, invasive disease may occur. Corticosteroids, antibiotics, and alterations in gastric $\mathrm{pH}$ predispose to invasive disease. ${ }^{6}$

\section{"Phagocytosis by neutrophils is an important part of the} host defences against infection with Candida spp"

In this case, the clinical presentation and the appearances of the bowel biopsy suggest that the gut was overgrown with $C$ albicans, which invaded the mucosa and penetrated the gut wall directly into the peritoneal cavity. This invasion may have been made possible by mucosal bacterial infection or ulceration from longterm non-steroidal anti-inflammatory drug treatment. Kopelson et al described two patients with intra-abdominal malignancy who developed isolated $C$ albicans peritonitis. They presumed that the tumour facilitated the passage of fungi into the peritoneum. ${ }^{7}$ Most cases of candidal peritonitis are documented in patients on chronic ambulatory peritoneal dialysis where there is direct inoculation through the skin.

Cases of spontaneous peritonitis with $C$ albicans are rare. ${ }^{8}$ This case report appears to be the first spontaneous $C$ albicans peritonitis in Felty's syndrome, and illustrates that the diagnosis may not be clear at the initial presentation. The case also demonstrates the difficulty in clearing these infections. Although the immediate cause for his death was ischaemic heart disease, the disseminated candidosis and methicillin resistant Staphyloccus aureus infection made an important contribution to the patient's morbidity. In view of 

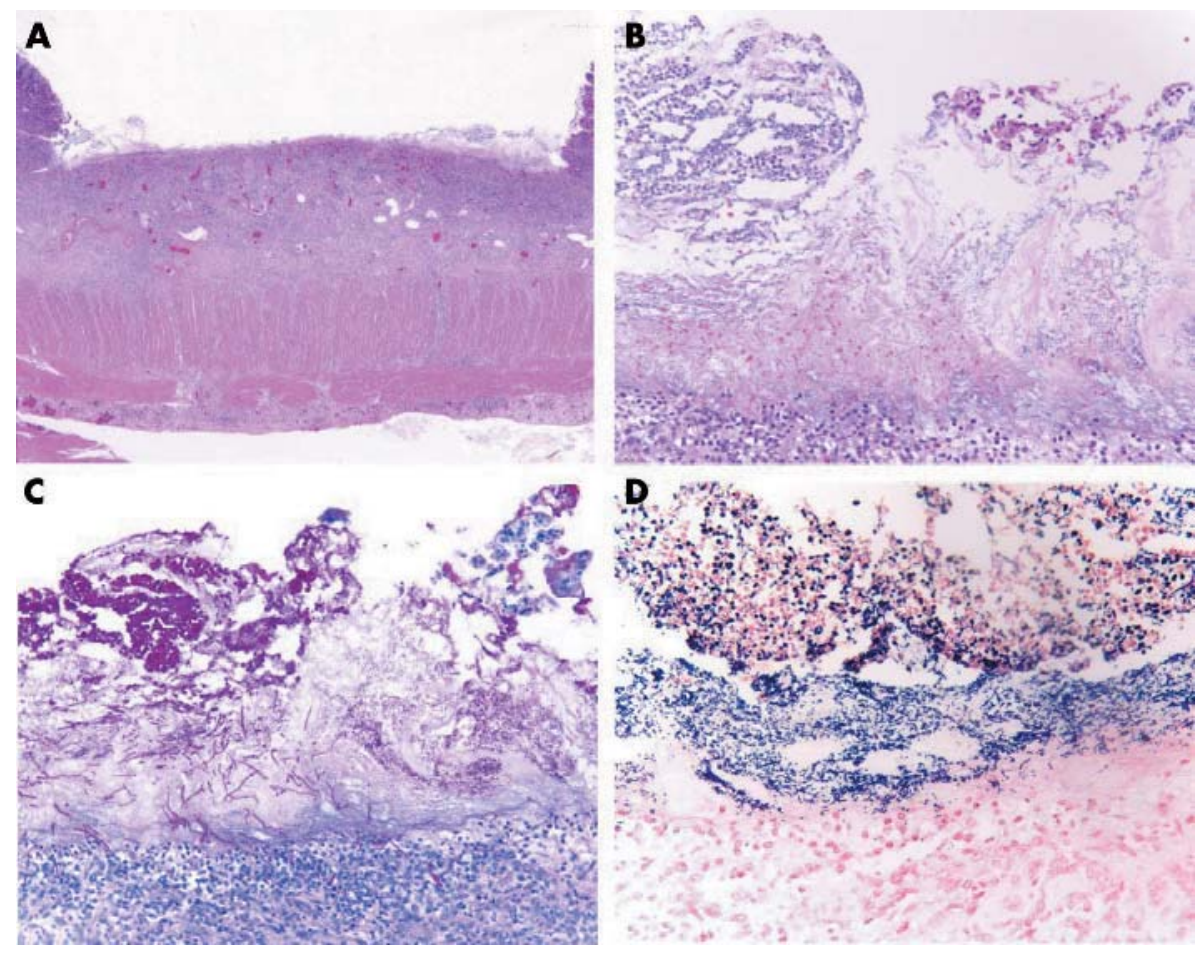

Figure 1 Microscopy of ulcer base. (A) Ulcer base of small bowel showing some fungal hyphae (haematoxylin and eosin (H\&E) stain); (B) edge of ulcer base exudates containing fungal spores and hyphae occasionally penetrating the granulation tissue (H\&E stain); (C) ulcer exudates clearly showing dense fungal spores and penetrating hyphae, invading deep into the granulation tissue (periodic acid Schiff stain); (D) Gram stain of ulcer base showing Gram positive cocci and occasional fungal spores.

\section{Take home messages}

- This is the first report of a patient with Felty's syndrome and spontaneous Candida albicans peritonitis

- The diagnosis was not clear at the initial presentation and the infection persisted despite treatment with fluconazole

- Although the immediate cause for this patient's death was ischaemic heart disease, the disseminated candidosis and methicillin resistant Staphyloccus aureus infection made an important contribution to his morbidity

the initial poor response to intravenous fluconazole, it is not clear whether continued use of oral fluconazole would have altered the outcome.

\section{Authors' affiliations}

D W Thomas, P Munuswamy, K Adu-Poku, C S Holgate, P Hickling, A Lambert, A G Prentice, Departments of Haematology, Histopathology, Rheumatology, and Surgery, Derriford Hospital, Plymouth, PL6 8DH UK
Correspondence to: Dr D W Thomas, Department of Haematology, West Suffolk Hospital, Bury St Edmunds, Suffolk IP33 2QZ, UK; wayne. thomas@wsh.nhs.uk

Accepted for publication 1 September 2004

\section{REFERENCES}

1 Felty AR. Chronic arthritis in the adult associated with splenomegaly and neutropenia. Bulletin of the Hopkins Hospital 1924;35:16-20.

2 Thorne C, Urowitz MB. Long-term outcome in Felty's syndrome. Ann Rheum Dis 1982;41:486-9.

3 Stanworth SJ, Bhavnani M, Chattopadhya C, et al. Treatment of Felty's syndrome with the haemopoietic growth factor granulocyte colony stimulating factor (G-CSF). Q J Med 1998;91:49-56.

4 Mäkitte AA, Bäck L, Aaltonen L-M, et al. Fungal infection of the epiglottis simulating a clinical malignancy. Arch Otolaryngol Head Neck Surg 2003; 129:124-6.

5 Kohler JR, Fink GR. Candida albicans strains heterozygous and homozygous for mutations in mitogen-activated protein kinase signalling components have defects in hyphal development. Proc Natl Acad Sci U S A 1996;93:13223-8.

6 Bodey GP. Candidiasis pathogenesis, diagnosis, and treatment, 2nd ed. Raven Press, 1993.

7 Kopelson G, Silva-Hutner M, Brown J. Fungal peritonitis and malignancy. Report of two patients and review of the literature. Med Pediatr Oncol 1979;6:15-22

8 Yang C, Yeh CT, Hung CF, et al. Case report: spontaneous peritonitis caused by candida albicans. J Gastroenterol Hepatol 1999;14:1041. 